\title{
La recezione del Petrarca nella poesia musicale della sua epoca: alcuni esempi
}

\author{
Francesco Facchin
}

\begin{abstract}
Il testo affronta il problema del rivestimento polifonico della produzione madrigalistica petrarchesca all'interno del contesto culturale relativo alla ricezione della poesia musicale dall'umanesimo al Rinascimento.
\end{abstract}

Parole chiave: Petrarca, musica, polifonia, madrigali.

\section{Abstract}

The text addresses the question of the polyphonic layering in Petrarchan madrigal production within the cultural context relating to the reception of musical poetry from Humanism through to the Renaissance.

Key words: Petrarch, music, polyphony, madrigals.

Multiforme e complesso è il rapporto che coinvolge Francesco Petrarca nella musica, non solo intesa come "nobile ricreazione», ma anche come scienza. Ne sono testimonianza le amicizie, gli scritti e i libri a lui appartenuti o che presumibilmente hanno avuto circolazione all'interno dell'ambiente culturale a lui legato. Da tempo sono noti i testi, pochissimi, del Petrarca messi in musica nella sua epoca: Non al suo amante più Dïana piacque, tuttavia, è il solo madrigale, entrato poi nei Rerum vulgarium fragmenta, del quale ci resti un' intonazione musicale, e polifonica, composta da Jacopo da Bologna - non è pervenuta invece la versione di Giovanni da Firenze. ${ }^{1}$ È stata, e a tutt'oggi è,

1. Giuseppe VecCHI, «Melica medievale, "Ars Nova» e lirica del Petrarca», in Dulce Melos, I, Bologna: A.M.I.S., 1972, p. 211-221: p. 217. Per le ipotesi sulla data di composizione (negli anni compresi tra 1348-1352) si vedano Giuseppe CoRSI, «Madrigali inediti del Trecento", Belfagor, Rassegna di varia umanità, n. XIV, 1959/1, p. 2-12: 7; poi approfondita da Pierluigi PETROBELLI, "Un leggiadretto velo" ed altre cose petrarchesche», in L. BiANCONI, F. A. Gallo, A. LanZa, L. LoockWood, P. Petrobelli (a cura di), Studi in onore di Nino Pirrotta, Rivista Italiana di Musicologia, n. X, 1975, p. 32-45: 35-40, in particolare p. 40. 
anche una tra le composizioni poetico-musicali più investigate dagli studiosi tanto di ambito letterario, quanto musicologico. ${ }^{2}$ In verità anche per altri testi, seppure assai parzialmente, è nota un'intonazione polifonica, ma essi appartengono all'area della "citazione» più o meno estesa; penso alla ballata di Bartolino da Padova Amor che nel pensier mio viv'e regna, primo verso del sonetto $R v f 140^{3}$ e al madrigale di Andrea Stefani Morte m'a sciolt'Amor d'ogni tuo legge la cui prima terzina corrisponde al congedo della Canzone Amor, se vuoi ch'io torni al giogo antico (Rvf 270). ${ }^{4}$ Altrettanto risapute sono alcune contenute intemperanze del poeta nei confronti dei postulanti interessati ad ottenere testi da recitare e, forse, da cantare; così come le testimonianze relative alla richiesta di suoi testi per dar loro "vestito» musicale. ${ }^{5}$ A questo riguardo sorprende l'espressione di Francesco di Vannozzo «[...] porgate del bel stil bello e polito» indirizzata a Petrarca nel sonetto Poi ch'a l'ardita penna la man diedi proprio per perorare la richiesta del musico Confortino atta ad ottenere versi da porre in musica. ${ }^{6}$ Meraviglia perché l'espressione, nonostante la ridondanza «bel stil

2. Laura PAOLINO, "Ancora qualche nota sui madrigali di Petrarca ("RVF" 52, 54, 106, 121)», Italianistica, n. XXX/2, Maggio-Agosto, 2001, p. 307-323; Marco GOZZI, «Il rapporto testo-musica nel madrigale di Petrarca «Non al so amante» musicato da Jacopo da Bologna», Kronos, n. III, 2001, p. 19-44; Stefano CAMPAGNOLO, "Petrarca e il Trecento musicale», relazione al Convegno «Petrarca in musica», Arezzo, 18-20 marzo 2004.

3. Paris, Bibliothèque nazionale de France, fonds nouv. acq. français 6771 , fol. $18 r$; la medesima struttura metrica fu ripetuta da Bartolino ne Linvido per lo ben che in altrui vede (unicum in Squarcialupi, fol. 116v).

4. Stefano CAmPagnOlO, "Petrarca e il Trecento musicale...» cit. In passato si è anche erroneamente ipotizzato che il madrigale tritestuale La fiera testa che d'uman si ciba — che nella silloge parmense 1081 (c. $91 v$ ) è rubricato come «Madrigale di M.F.P.»— fosse da assegnare al Poeta. Cfr. Ireneo AfFò, Dizionario precettivo, critico ed istorico della Poesia volgare, Milano: Silvestri, $1824^{2}$ [1777], ad vocem «Madrigale», p. 235-237 (rist. con Introduzione e indici a cura di Franca MAGNANI, Università degli Studi di Parma, Istituto di Filologia Moderna, Forni, 1993); Rime disperse di Francesco Petrarca o a lui attribuite, per la prima volta raccolte a cura di Angelo SOLERTI, ed. postuma con prefazione, introduzione e bibliografia, Firenze, Sansoni, 1909, p. 259 [ristampa in facsimile, Firenze, Le Lettere 1997]; cfr. Giuseppe VECCHI, "Melica medievale», cit., p. 216-217; Giuseppe CORSI (a cura di) Poesie musicali del Trecento, Bologna: Commissione per i testi di lingua, 1970, p. 96-97; Cristina ZAMPESE, «I madrigali di Franco Sacchetti», ACME: Annali della Facoltà di Lettere e Filosofia dell'Università degli studi di Milano, n. XXIV/2, Maggio-Agosto 1981, p. 373-386: 383; Gianluca D’AGOSTINO, "La tradizione letteraria dei testi poetico-musicali del Trecento: una revisione per dati e problemi. (L'area toscana)", in Col dolce suon, che da te piove. Studi su Francesco Candini e la musica del suo tempo, in memoria di Nino Pirrotta, a cura di A. Delfoe, M. T. Rosa Barezzani, SISMEL, 1999. cit., p. 389-428: 413. L'autore dà un quadro della principale bibliografia relativa alla fonte letteraria nonché gli aspetti relativi alle varianti letterarie tra le due edizioni musicali.).

5. Si veda la lettera a Giovanni Boccaccio, Sen. V, 2. Cfr. Alessandro PANCHERI, «Pro Confortino", in Cesare SEGRE, Le varianti e la storia. Il canzoniere di Francesco Petrarca con due interventi di Giovanni Giudici e Alessandro Pancheri, Torino, 1999, p. 51-53; Lettere senili di Francesco Petrarca volgarizzate, Firenze 1869-1870, trad. di G. Fracassetti.

6. Padova, Biblioteca del Seminario, cod. 59, f. 11: Poi ch'a l'ardita la man diedi, sonetto di Francesco di Vannozzo indirizzato a F. Petrarca per invitarlo a scrivere nuovamente versa da porre in musica per un tal Confortino. 
bello", resta equivoca e dunque è da chiarire nel suo valore e significato che sembrerebbe indirizzare verso un'accezione più tecnica che estetica. Non entrerò nel merito di questi luoghi, piuttosto mi soffermerò su un fenomeno che interessa l'opera di Petrarca nel processo di circolazione nell'ambito della poesia per musica e che vede componimenti come il sonetto Amor che nel pensier mio vive regna trasformarsi, con una sostanziale e profonda elaborazione, nella corrispondente ballata.
A. Amor che nel pensier mio viv'e regna
B. e 'l suo seggio maggior nel mio cor tene,
B. talor armato ne la fronte vene;
A. ivi si loca et ivi pon sua insegna

A. Quella ch'amare e sofferir ne 'nsegna,
B. e vol ch 'l gran desio, l'accesa spene,
B. ragion, vergogna e reverenza affrene,
A. di nostro ardir fra se stessa si sdegna.

C. Onde Amor paventoso fugge al core, D. lasciando ogni sua impresa, e piange e trema;

C. ivi s'asconde e non appar più fore.

C. Che poss'io far, temendo il mio Signore, D. se non star seco infin a l'ora estrema? C. ché bel fin fa chi ben amando more.
Z. Amor che nel pensier mio viv'e regna

Z. d'est'alta donna sopra ogn'altra insegna

A. Amor, Amor ogni pensier m'enduce

B. con dolce affanno ben servire amore.

A. però che d'ogne nobeltà traluce

B. la donna in vista fonte de valore.

B. Donca ben piaza al mio degno segnore

Z. che mi conservi in questa donna degna.

Neri, 1983

$\mathrm{Al}$ di là dei parallelismi di parole-rima e di concetti, non passa inosservato l'isomorfismo strutturale dell'ultimo verso, indizio significativo per ammettere che ben più di semplice citazione possiamo parlare, piuttosto di alta tecnica di elaborazione compositiva che sfrutta richiami(?) e relazioni a carico della memoria per rendere vivo e nuovo(?) il ricordo di un modello poetico. Tanto meno non sfugge il legame che sottende un gruppo di madrigali ispirati al sonetto Passa la nave mia colma d'oblio (Rvf. 189), dove si assiste al processo di trasformazione di questa composizione nella forma del madrigale. Guido Capovilla ha evidenziato che, nel repertorio poetico musicale del Trecento, $\mathrm{i}$ madrigali di cui non ci è pervenuta intonazione né altra notizia sono circa una sessantina, per la maggior parte di «autore»; e tuttavia non poche sono le intonazioni andate perdute, così come, stando ad un esempio singolare qual è il canzoniere autografo di Franco Sacchetti, non poche attribuzioni a compositori sono poi cadute. ${ }^{7}$ 
Il sonetto del Petrarca si richiama com'è risaputo al quarto Salmo penitenziale; ma il tema della nave in balia degli elementi, del naufragio, il carattere meditativo di tale rappresentazione e i significati connessi alla caducità delle cose terrene, quindi della necessità di un "porto» dove trovare pace e sicurezza, non è nuovo all'interno della sua opera. ${ }^{8}$ La seconda strofe della canzone $R v f$ 323 Standomi un giorno solo a la fenestra ${ }^{9}$ contiene, ancora una volta, tutti gli elementi, sebbene inseriti in una forma esameronale e nel contesto della visione. L'elemento devastatore è il medesimo di natura meteorologica: la tempesta, nell'oscurità invernale della mezzanotte; le immagini sono tutte presenti con il loro valore talvolta di negatività, la nebbia, la nave, la vela e le sartie, il vento. ${ }^{10}$

A fronte delle due composizioni del Petrarca saranno di seguito analizzati i madrigali intonati rispettivamente da Donato da Cascia, Nicolò del Proposto e Paolo da Firenze: I'o perduto l'alber e 'timone, su testo di Rigo Belondi; ${ }^{11}$ Rott'e la vela, l'arbor e l'antenna ${ }^{12} \mathrm{e}$, su testo di Franco Sacchetti ma intonato ancora da Nicolò, Nel mezzo già del mar la navicella, ${ }^{13}$ madrigale che fu modello per un anonimo testo laudistico: Nel mezzo a duo ladron post'una stella; ${ }^{14}$ infine, Fra duri scogli sanz'alcun governo e Corse per l'onde già di speme piena. ${ }^{15}$ I musicisti, com'è noto, sono tutti personaggi presenti nella scena culturale fiorentina: Donato e Nicolò sono i più vicini a Petrarca essendogli contemporanei; e non è da escludere che il Donato e il Francesco nominati in un' epistola (Fam. XIV, 3) altri non siano che Donato da Cascia e Francesco Landini. Nicolò da Perugia (o del Proposto), ${ }^{16}$ assieme a Donato, fu tra i primi compositori ad into-

8. Francesco Petrarca, Canzoniere. Edizione commentata, nuova edizione aggiornata, a cura di Marco Santagata, Milano: Arnoldo Mondadori Editore, $2004^{2}$, p. 828-831.

9. Francesco Petrarca, Canzoniere, cit., p. 1242-1258.

10. Per un'analisi puntuale della canzone si veda Bertolo MARTINELLI, «Veduta con naufragio: «Rerum vulgarium fragmenta» CCCXXIII, 13-24», Italianistica. Rivista di letteratura italiana, n. XXI/1, gennaio/aprile 1992, p. 511-535.

11. Firenze, Biblioteca Medicea Laurenziana, ms. Palatino 87, cc. $76 v-77 r$ (codice Squarcialupi).

12. Firenze, Biblioteca Medicea-Laurenziana, Palatino 87, cc. $87 v-88 r$.

13. Paris, Bibliothèque Nationale Fonds italien 568, cc. $28 v-29 r$; Firenze, Biblioteca Nazionale Centrale, Panciatichiano 26, cc. 86v-87r; Firenze, Biblioteca Medicea-Laurenziana, Palatino 87 , cc. $81 v-82 r$; il solo testo è copiato in Firenze, Biblioteca Medicea-Laurenziana, Ashburnham 573, c. $9 r$ e Stato del Vaticano, Biblioteca Apostolica Vaticana, Chigiano L. VIII. 300, p. 300. Gianluca D’Agostino, La tradizione letteraria, cit., p. 389-428: 411. Per quanto concerne le relazioni stemmatiche tra le tre fonti che conservano Nel mezzo già del mar le concordanze tra Squarcialupi e Pit contro Panciatichi 26 rinvio a Eugene C. FELLIN, A Study of superious Variants in the Sources of Italian Trecento Music: madrigals and cacce, Ph.D. diss. Univ. of Wisconsin, 1970, 4 voll. p. 83, trascr.: p. 80-84; ID., "Le relazioni tra i manoscritti musicali del Trecento", Rivista Italliana di Musicologia, n. VIII/2, 1973, p. 165-180: 167.

14. Firenze, Biblioteca Riccardiana, ms. 2871, cc. 60v-61r. Giuseppe CorsI, «Madrigali inediti del Trecento", Belfagor, n. XIV, 1959/3, p. 1-12: 2.

15. Paris, Bibliothèque Nationale Fonds italien 568 , cc. $58 v-59 r$ e $59 v-60 r$.

16. Fu probabilmente un frate domenicano di Santa Maria Novella; per i suoi rapporti con compagnia di San Zanobi ebbe presumibilmente l'incarico del canto delle laude e dell'insegnamento della grammatica ai bambini. Per una discussione sulle ipotesi di identificazione di Nicolò si veda Blake MC D. WILSON, «Madrigal, Lauda, and Local Style in Trecento Florence», The Journal of Musicology, n. XV/2, 1997, p. 137-177: 148n. 
nare ballate polifoniche negli anni tra il 1360-1365. Paolo da Firenze (Paulus Abbas, Paolo tenorista), nato in territorio fiorentino nel $1355 \mathrm{ca}$., morì a Firenze, non prima del 21 settembre 1436 , data del suo testamento. ${ }^{17}$ Personaggio colto, Pirrotta ha ritenuto sia stato egli stesso l'autore dei testi intonati. ${ }^{18}$

Origini fiorentine hanno anche i codici che conservano queste composizioni: Pit (Paris, Bibliothèque Nationale, fonds italien, ms. 568), ${ }^{19}$ la più ampia silloge di composizioni di Paolo, fu copiato probabilmente nello scriptorium di Santa Maria degli Angeli, come il codice Squarcialupi (Firenze, Biblioteca Medicea Laurenziana, ms. Pal. 87). Le datazioni sono comprese tra gli anni $1385 / 1390$ con aggiunte tra gli anni $1420 / 1430$ per il Panciatichi $26 ;^{20}$ 1405/1405-1408 per Pit; ${ }^{21}$ agli anni 1410-1415 ca. per lo Squarcialupi. ${ }^{22}$

Un primo raffronto quale si può rilevare dagli schemi (Tav. 1) dei singoli madrigali mette già in evidenza le diverse dipendenze e rapporti di maggiore o minore vicinanza con il sonetto del Petrarca.

Le due composizioni di Paolo Fra duri scogli e Corse per l'onde hanno entrambi uguale schema ABA BCB CC a rime incrociate di derivazione dantesca - tra i pochi schemi utilizzati da Petrarca - e corrisponde allo stesso utilizzato per il madrigale Non al suo amante. Guido Capovilla ne ha segnalato la relativa rarità: ricorre solo sei volte nella letteratura madrigalistica dell'Ars nova del Trecento. ${ }^{23}$

17. Diventò monaco benedettino attorno al 1380 ca.; ebbe più incarichi religiosi: prima abate di San Martino al Pino, fu quindi amministratore dei beni di Santa Maria degli Angeli a Firenze. Cfr. Biancamaria BrumanA-Galliano CiliberTI, "L'opera di Paolo da Firenze in una nuova fonte di Ars Nova italiana», in Luigi PesTALOZZA (a cura di), La musica al tempo di Dante, Musica/Realtà, 1986 (Atti del Convegno Opera di Dante, Comune di Ravenna, 1214 settembre 1986), p. 198-205:202.

18. cfr. John NÁDAS, The Songs of Don Paolo Tenorista: The Manuscript Tradition, in In cantu et in sermone: A Nino Pirrotta nel suo $80^{\circ}$ compleanno, a cura di Fabrizio Della Seta e Franco Piperno, Firenze, 1989, p. 41-64: 45, 55.

19. Per uno studio sulle datazioni del manoscritto proposte da Nino Pirrotta, Kurt von Fischer e Gilbert Reaney si veda John NÁDAS, The Transmission of Trecento Secular Polyphony: Manuscript Production and Scribal Practice in Italy at the End of the Middle Ages, Ph. D Dissertation, New York University, 1985, p. 218-222.

20. Per una più recente analisi del manoscritto e datazione si veda Stefano CAMPAGNOLO, Il codice Panciatichi 26 della Biblioteca Nazionale di Firenze nella tradizione delle opere di Francesco Landini, in Col dolce suon, cit., p. 77-119: 108-114.

21. Per una descrizione del processo di compilazione e della cronologia del manoscritto si veda John NÁDAS, The Songs of Don Paolo, cit., p. 50-64.

22. Per una discussione sulle ipotesi di datazione si veda John NÁDAS, «Il codice Squarcialupi: una 'edizione della musica del Trecento (ca. 1410-1415)» in F. Alberto Gallo (a cura di) Il codice Squarcialupi. MS. Palatino 87, Biblioteca Medicea Laurenziana di Firenze, FirenzeLucca: Giunti Barbèra-Libreria Musicale Italiana, 1992, p. 23-28.

23. Thomas Marrocco, «The Fourteenth-Century Madrigals: its Form and Contents», Speculum, n. XXVI/3, 1951, p. 449-457: 450-451; Guido CAPOVILla, Materiali, cit., p. 235: schema VII. È da notare come Paolo utilizzi tale modello tre volte su sei attestazioni complessive. Non sfugga che tale schema con una strofa in più: ABA BCB CDC EE (schema VI in ID., p. 235), che ricorre una sola volta fu usato da Francesco Landini per il madrigale $S i$ dolce non sonò con lira Orfeo, cfr. MEMELSDORfF, La «Tibia» di Apollo, cit., p. 250-253 e in particolare p. 253 dove vi è il raffronto sotto il profilo formale delle parole-rima con i Rerum vulgarium fragmenta. 
Il madrigale di Nicolò/Sacchetti Nel mezzo già del mar, con l'anonimo travestimento spirituale Nel mezzo a duo ladron, usa il modello ABB ACC DD - da Petrarca utilizzato in Or vedi, Amor, che giovinetta donna- ricorre ventidue volte. ${ }^{24}$ Infine i due madrigali Rott'e la vela di Nicolò e I' ho perduto di Donato/Belondi presentano entrambi lo schema ABB CDD EE che nel repertorio metrico vanta ottantuno esempi codificati e sette varianti. ${ }^{25}$

In Paolo il rapporto con il sonetto del Petrarca è ben leggibile nel madrigale Fra duri scogli non solo nel richiamo incrociato di parola e rima nella prima terzina: verno - governo (2-3v) contro governo-verno $(1 / 3 \mathrm{v})$ ma anche nell'inversione della figura nel secondo verso: «per aspro mare, a mezza notte il verno" di contro a "mi trovo lasso! in tempestoso mare». ${ }^{26}$ Il madrigale Corse per l'onde già di speme piena, invece, nel senso generale sembra potersi appaiare alla seconda strofa Indi per alto mar vidi una nave (v. 13-24) della canzone Standomi un giorno solo a la fenestra (Rvf 323), seppure non manchino parallelismi anche con il sonetto. ${ }^{27}$ In entrambi si contrappone una situazione positiva: da una parte una nave sta solcando con il sartiame di seta e le vele d'oro un «mar tranquillo» ma viene travolta da un'improvvisa tempesta da oriente che la spinge su uno scoglio e la trasforma in una stretta bara che nel profondo nasconde ricchezze non più superate (Petrarca); dall'altra la nave, che "corre» tra le onde sicura di trovare un «buon porto», viene invece spinta nella tempesta proprio alla fine del "giovin tempo", da un "nuov'uccello» che scende dal cielo a portar via ogni speranza. ${ }^{28}$

Per i testi di Nicolò, Eugene C. Fellin ha mostrato una stretta relazione tra le fonti che conservano il madrigale Nel mezzo già del mar, in particolare Panciatichi 26 e Pit in rapporto alle varianti musicali con Squarcialupi. ${ }^{29}$ Entrambi i madrigali Nel mezzo già del mar e Rott'è la vela mostrano frequenti condivisioni non solo lessicali ma anche di metafore sia con il sonetto del Petrarca sia, trasversalmente, con la seconda strofa della canzone Rvf. 323, sebbene in questi casi, ma soprattutto nel secondo dei due madrigali, il senso dato è di difesa dall'Amore non corrisposto «in questo mar, Amor, più non mi cogli». Si osservi anche la piccola ma efficace consonanza a cavallo della cesura dell'ultimo verso: mar, Amor, l'uno quasi l'anagramma dell'altro; richiama l'analogo artificio sarte di seta usato dal Petrarca nel secondo verso della seconda stanza della canzone Indi per alto mar vidi una nave (Rvf 323).

24. Guido CapOvILLA, Materiali, cit., p. 237: schema XXX.

25. Ibid, cit., p. 239-240: scema XLVIII; utilizzato dal Sacchetti quindici volte.

26. Ibid, Morfologia, cit., p. 201; Per un'analisi sui testi di Paolo si veda in Giovanni CARSANIGA, «I testi di Paolo Tenorista (Nuove proposte di lettura)», Studi e problemi di critica testuale, n. 40, 1990, p. 5-22.

27. Francesco Petrarca, Canzoniere, cit., p. 1242-1244 e 1248-1249.

28. Per una riflessione sui due madrigali di Paolo cfr. Giovanni CARSAniga, I testi di Paolo, cit. p. 22.

29. Eugene FELLIN, A Study of superious Variants, cit. passim; e ID., Le relazioni tra i manoscritti musicali, cit., 167-168. Si veda anche Cristina ZAMPESE, Il Libro delle rime di Franco Sacchetti, MA diss., Università di Milano, a.a. 1979-1980. 
L'immagine della tempesta che in Petrarca ricorre al sesto verso «che la tempesta e 'I fin pare ch'abbi a scherno" si ritrova nel primo verso della seconda terzina in Nicolò "col vento tempestoso e quella stella»; immagine che parallelamente si ritrova alla medesima altezza in Rott’e la vela con il verso «Coverta m'è la tramontana stella». Nicolò anticipa così il senso pessimistico espresso nell'ultimo verso del sonetto: la stella (la tramontana stella: Venere $=$ amore) lo ha reso fedele e così più velocemente «afretta sua giornata, e la mia morte». In Rott'e la vela il secondo verso "del mie navili, ond'aprodar non posso" richiama l'immagine conclusiva del sonetto «tal ch'incomincio a desperar del porto».

Il madrigale intonato da Donato da Cascia I'ò perduto l'alber non manca di presentare parallelismi di lessico, immagini e concetti, uno per tutti: gli ultimi versi «E non ò isperanza né conforto | d'aver bonaccia o mai riaver porto» corrisponde all'ultimo del sonetto petrarcesco.

Sebbene al raffronto trasversale delle immagini espresse dai diversi testi con il sonetto le metafore del Petrarca rivelino una maggiore eleganza di espressione e sottigliezza di senso e significati, ritroviamo nei madrigali un susseguirsi, anche se in posizioni diverse, di analoghe espressioni, secondo una simmetrica scansione logico-sintattica anche se il senso più intimo è spesso "alleggerito» nella sua drammatica e angosciosa inquietudine esistenziale rispetto al sonetto.

Nicolò e Donato hanno mantenuto un legame con il sonetto attraverso le immagini e parzialmente nel lessico, solo il testo di Franco Sacchetti condivide un medesimo schema di rime utilizzato anche dal poeta. Viceversa Paolo mostra un'intenzione e un'attenzione particolare a questo genere di richiami con la condivisione di schema delle rime e, parole-rima. Ma Paolo non è nuovo a questi "recuperi», già nel madrigale Non più 'nfelice a le suo membra nacque ${ }^{30}$ aveva utilizzato la medesima struttura di Non al suo amante più Dïana piacque con il rapporto chiastico delle parole-rima della prima terzina: nACQUE - PIACQUE contro PIACQUE - ACQUE. ${ }^{31} \mathrm{Ma}$, la consapevolezza di Paolo va oltre al prestito testuale - e tale consapevolezza è esplicitata dal testo del madrigale Se non ti piace ${ }^{32}$ _; nel caso specifico di Non più 'nfelice già John Nádas aveva rilevato anche per la musica lo stretto rapporto con

30. Madrigale ha lo stesso capoverso di una ballata cfr. Bianca BECHERINI, "Antonio Squarcialupi e il codice Mediceo Palatino 87», in Bianca BECHERINI (a cura di), L'Ars nova italiana del Trecento, Certaldo: Centro di Studi sull'Ars nova italiana del Trecento, 1962, (Primo Convegno Internazionale 23-26 luglio 1959), p. 141-196: 167-168.

31. Si veda in Guido CaPOVILla, Materiali, cit., p. 200-201. Per un ulteriore approfondimento esegetico e di una probabile filogenesi di questo madrigale in rapporto agli altri tre, ma anche in rapporto alla canzone $R v f 323$, che come vedremo ha, a sua volta, contatti non occasionali con il sonetto $R v f 189$, si veda Laura PAOLINO, 'Ancora qualche nota, cit., p. 322-323.

32. Tale consapevolezza è esplicitata dal testo del madrigale Se non ti piace, che in Pit segue immediatamente Non più 'nfelice (cc. 34v-35r e 35v-35r-36r) dove Paolo deplora quei compositori che denigrano i predecessori che resero possibili queste opere; e tale ingratitudine gli richiama alla mente quegli angeli ribelli condannati da Dio. Cfr. John NÁDAS, Song Collections, cit. p. 132. 
l'intonazione di Non al suo amante: Paolo renderebbe omaggio a Jacopo da Bologna, compositore appartenente alla prima generazione di polifonisti del Trecento. $^{33}$

Nel complesso, anche osservando lo schema delle ricorrenze lessicali e sintattiche (Tav. 2), emerge il diverso rapporto che i cinque madrigali intonati hanno con il testo del Petrarca. Se sul fronte delle ricorrenze lessicali e sintattiche i testi di Donato e Nicolò denunciano una maggiore presenza di immagini e concetti paralleli al sonetto del Petrarca, già Franco Sacchetti ne riduce la quantità e trasforma il lessico pur nella persistenza dell'immagine. I testi di Paolo, infine, scarnificano all'essenziale la citazione lessicale mostrando invece un interesse maggiore per quelle caratteristiche che rinviano alla forma (anche «a distanza» com'è il caso di sonetti «recuperati» in madrigali) e alla struttura metrica, magari attraverso richiami incrociati che tanto, ad un musicologo, rinviano alla tecnica dello stimmtausch utilizzata nelle prime forme polifoniche: ossia dello scambio tra le voci di due diversi elementi melodici tra loro contigui seppure alternati nella giustapposizione seriale. Ciò può avere una motivazione che si rapporta al ruolo e alla stessa ricezione della poesia di Petrarca e al nascente fenomeno del «petrarchismo»: per i contemporanei Petrarca è sì un maestro ma, forse, ancora interessano maggiormente gli atteggiamenti più esteriori della poesia, ossia le immagini che la sua attenzione e ricchezza lessicale suggeriscono e evocano. In questa fase è un'eccezione il madrigale di Jacopo da Bologna Non al suo amante più Dïana piacque, e si può spiegare solo attraverso gli altri testi intonati dal musicista. In assonanza con Jacopo è Paolo da Firenze, ma con lui ci troviamo anche in un momento più avanzato di penetrazione e riconoscimento dell'opera poetica del Petrarca. Essa rappresenta già un modello formale e di tecnica nell'uso della metrica, come tale viene riconosciuta e ricalcata. Paolo dunque, compie presumibilmente una duplice operazione di omaggio: a Jacopo, come già osservato e a Petrarca riutilizzandone gli elementi più profondi e intimi del suo poetare, non esplicitamente ma nel costante richiamo alle stesse modalità compositive e immaginifiche che il Poeta aveva con tanta raffinatezza reso accessibili.

Sotto il profilo musicale i madrigali mostrano, accanto alle caratteristiche proprie di ciascun autore, elementi comuni e comportamenti condivisi che potrebbero configurarsi come altrettanti rimandi: elementi formali, lessicali e sintattici che sono relativi a esperienze di tipo intellettuale nelle quali il rapporto con il testo sembra essere più speculativo che emotivo. Tale osservazione non esclude, peraltro, che l'attuale ottica possa essere determinata anche dalla distanza temporale che ci separa e che ciò abbia allentato i legami «semantici» percepiti, e riconosciuti, all'epoca.

33. cfr. John NÁDAS, "Song Collections in Late-Medieval Florence», in Trasmissione e recezione delle forme di cultura musicale. Round Tables II: Costituzione e conservazione dei reertorii polifonici nei secoli XIV e XV (Atti del XIV Congresso della Società internazionale di Musicologia, Bologna 27 agosto - 1 settembre 1987, Ferrara - Parma, 30 agosto 1987), Torino: EDT, 1990, p. 126-137: 132-133. 
Per quanto attiene la sezione del «ritornello» la forma è rispettata, pur se con alcune varianti, solo da Paolo, il più giovane dei compositori qui citati. Nel madrigale Fra duri scogli la melodia del superius si ripete due volte —con tenor diversificato per ciascuna volta - per intonare i due versi di cui il ritornello si compone, ${ }^{34}$ mentre in Corse per l'onde l'intonazione figura una sola volta, ma conclusa dalle due cadenze di «verto» per il primo verso e «chiuso» per il secondo.

Vale forse ricordare che, nelle fonti manoscritte, il testo poetico giustapposto alla musica è solo quello relativo al primo terzetto, mentre il rimanente è trascritto nel residuum; e così anche per il ritornello, tranne nei casi nei quali è interamente intonato. I singoli versi del terzetto ricevono ciascuno una diversa melodia scandita da vocalizzi sulle sillabe iniziale e penultima di ogni verso; talvolta sul secondo verso, o sul terzo, vi è un cambio di tempo. Sinteticamente lo schema generale, come di consueto, risulta:

$\begin{array}{lrccc}\text { Musica: } & \alpha \beta \gamma & \alpha \beta \gamma & \delta & \delta \\ \text { Testo: } & \text { I terzetto } & \text { II terzetto } & \begin{array}{c}\delta \\ \text { I verso } \\ \text { ritornello }\end{array} & \text { II verso }\end{array}$

Nella prima sezione relativa alle terzine di Fra duri scogli l'inciso anacrusico che appare all'apertura del primo verso (mis. 10) ${ }^{35}$ si ripete variamente modificato anche nel melisma conclusivo (Es. 1); riprendendo il profilo melodico che scandisce le prime sette note del melisma iniziale sulla prima sillaba (mis. 3). L'inciso ritmico iniziale del secondo verso (miss. 25-26) appare come uno sviluppo dell'elemento ritmico presente nella terza misura, ${ }^{36}$ anticipato già nel tenor (miss. 22-23) ma con l'inversione dei due elementi che lo costituiscono (Es. 2). Anche questa nuova unità si ripete sostanzialmente due volte: la seconda con imitazione canonica tra tenor e superius (miss. 29-30). L'intonazione che caratterizza il terzo verso, infine, compenetra l'incipit anacrusico della prima frase con la figurazione sincopata che caratterizza l'andamento ritmico della seconda (Es. 3); e, ancora una volta, il motivo è ripetuto due volte (mss. 40-41 e 46-47 incipit con var).

Si segnala dunque che, se nella macrostruttura di ogni sezione i temi ritmico-melodici si presentano, pur con trasformazioni, spesso due volte in una sorta di allitterazione, ciò avviene anche a livello di microstruttura dove il medesimo inciso è presentato a coppie.

Con maggiore evidenza stessi motivi — più o meno variati, anche per aumentazione o retrogradazione- si ripetono nella voce superiore (il supe-

34. Comportamento già segnalato da John NÁDAS, «The song of Don Paolo Tenorista: the manuscript tradition", in Fabrizio DELLA SETA-Franco PIPERNO (a cura di), In Cantu et in Sermone. For Nino Pirrotta on his 80th Birthday, Firenze: Olschki-Univ. of W. Australia Press, 1989, p. 41-64: 59.

35. per la numerazione delle misure si prendono le trascrizioni fornite dall'edizione dell'Oiseau Lyre, voll. VII, VIII, IX (Polyphonic Music of the Fourteenth Century).

36. si veda l'Es. 1 . 
rius) all'interno di ogni sezione nel secondo madrigale di Paolo, Corse per l'onde (Es. 4): dall'inciso di apertura corrispondente al lungo vocalizzo sulla prima sillaba Cor- (mis. 2-4, 9-10) e in modo simile, per retrogradazione, alle parole (la) navicella (miss. 24-25, 27-28). Ugualmente con variazioni ritmiche al vocalizzo sulla penultima sillaba del primo verso: pie-[na] e nel secondo verso alle parole d'aver buon por-[to] (miss. 16-19, 30-34) e per aumentazione (Es. 5), nel vocalizzo iniziale e finale del terzo verso (Die)[tr'al], e (se- $\underline{r e})-[n a]$ (miss. 43 e 50). Queste melodie talvolta assumono l'apparenza di vere e proprie "progressioni» anche «armoniche» (Es. 6) come ai versi (Die-) tr'alla stel- [...] —la lucid'e (miss. 44-48), (Ma) nuov'uccel / per l'aria giù (miss. 75/79); e nel vocalizzo sulla sillaba finale del ritornello (miss. 81/85).

Non mancano tra i due madrigali di Paolo taluni elementi ritmici ricorrenti che costituiscono quasi dei termini di raccordo e continuità lessicale e stilistica (Es. 7). Se nelle due composizioni di Paolo i comportamenti descritti appaiono essere molto strutturati e sembrerebbero raccordarsi al testo poetico, ciò nondimeno analoghe condotte nell'elaborazione del materiale interno sono reperibili anche nei due madrigali di Nicolò. Anzi, nel primo: Nel mezzo già del mar è particolarmente evidente il riutilizzo tanto del medesimo materiale tematico (Es. 8), quanto di richiami tra le parti conclusive dei melismi al temine del primo e del terzo verso delle terzine e gli incipit dei due distici del ritornello (Es. 9a,b). Parimenti, estesi sono i rinvii ad analoghi modelli ritmico-melodici in Rott'e la vela (Es. 10): dai movimenti d'apertura dei melismi con i quali iniziano il primo e terzo verso delle terzine, all'insolita ripetizione del terzo verso stesso (Es. 11a,b). ${ }^{37}$

Nel madrigale di Donato I'ò perduto l'alber' condiviso è sempre lo sviluppo costruttivo: medesime figurazioni ritmico-melodiche sono utilizzate con varianti interne (Es. 12) e richiami sono presenti anche nel tenor (Es. 13) giustapposti a differenti motivi del superius. Con la composizione di Nicolò ha in comune sia l'uso della Longa — nel tenor una Maxima - al principio e al termine delle prime due sezioni che scandiscono i corrispondenti versi delle terzine sia, nel ritornello, l'aver composto per esteso l'intonazione corrispondente ai versi del distico. Al contrario, Paolo, come già s'è detto, non intona per esteso i due versi del ritornello e, se pure mantiene la medesima melodia al superius per il secondo verso (Fra duri scogli), modifica tuttavia il tenor.

Singolari risultano alcune consonanze che corrono trasversalmente da Donato e Nicolò a Paolo: sorta di duplicazione musicale di altrettanti parallelismi testuali. È il caso dell'elemento in sincope accompagnato da quartina al tenor

37. (miss. 76/81 _ 85/89). Per più approfondite ricerche sulla ripetizione di parole cfr. Agostino ZIINO, «Ripetizione di sillabe e parole nella musica profana italiana del Trecento e del primo Quattrocento: proposte di classificazione e prime riflessioni», in Ursula GÜNTHER - Ludwig Finscher (a cura di), Musik un Text in der Meherstimmigkeit des 14. und 15. Jahrhunderts, Vorträge des Gastsymposions in der Herzog August Bibliothek Wolfenbüttel, 8. bis 12. September 1980, Kassel - Basel - London: Bärenreiter, 1984, p. 93-119. 
(Es. 14) che in Nicolò (Rott’e la vela) intona l'arbore l'anten(na) (mis. 15) e poi si trasforma in semplice quartina con l'introduzione di una pausa variamente permutata nella posizione (miss. 76/78, 84/85); in Paolo (Fra duri scogli) intona (tro-) vo lasso in tempestoso; (Per-)duto ho l'orza e son a mezo (miss. 29/31, 40/43) e (Cor-se per l'onde) Corse per l'onde (miss. 5, 11). Ma anche l'elemento melodico-ritmico discendente con inizio anacrusico che, in entrambi gli autori, caratterizza il melisma conclusivo di ciascun verso (Es. 15). Il secondo madrigale di Nicolò Nel mezzo già del mar condivide con Fra duri scogli il tempo del ritornello e il motivo della testa del tema iniziale con le successive tre note permutate (Ess. 16 e 9), oltre ad una altrettanto singolare analogia ritmica delle prime misure nel tenor.

Quanto a Donato le prime dieci misure del madrigale I'o perduto, mostrano sorprendenti simmetrie con le corrispondenti misure del madrigale $\mathrm{Nel}$ meço di Nicolò (Es. 17). Elementi ritmici ricorrenti, pur con inevitabili variazioni di diversa entità, si possono rinvenire in più momenti all'interno dei brani qui presentati (Nicolò: miss. 7-9, 14-16, 18-21, 24-26 e 22-27 = 6370var.; Donato: miss. 7-8, 9-10, 18-19). Con Paolo (Corse per l'onde), il madrigale di Donato spartisce, seppure episodicamente, il motivo discendente a note ribattute (Es. 18); in entrambi intona il terzo verso in tempo ternario ('senaria italica').

Alberto Gallo già segnalò i comportamenti fin qui descritti inscrivendoli nelle figure retorico-musicali dell'anafora, la ripetizione di una medesima frase musicale e del sonus ordinatus, il motivo sui gradi contigui discendenti di una scala; figure, per altro, rilevate anche nella produzione di Jacopo da Bologna. ${ }^{38}$ Precedentemente, Thomas W. Marrocco aveva ricondotto questi procedimenti all'interno del repertorio tràdito dai più antichi frammenti di Ostiglia — parte del Codice Vaticano Rossi 215 - segnalandone nel contempo anche la rarità data la loro presenza nel solo madrigale anonimo E com' chavalché el cont' Ugo per la strada, composizione che presenta particolarità sotto il profilo formale essendo priva del ritornello. ${ }^{39}$

L'interesse dei tre autori, Nicolò, Donato e Paolo per la poesia petrarchesca ha in definitiva sortito dei risultati affatto diversi che emergono sotto i profili dell'approccio nell'elaborazione e interpretazione poetica e musicale. Solo Paolo sembra aver prestato attenzione ad evidenziare un legame anche, ma non solo, formale con il testo del Poeta. Donato e Nicolò sembrano fermarsi più all'aspetto immediato che il lessico e le immagini suggerite rimandano e in particolare per Nicolò, forse ad una maggiore concentrazione per la forma musicale, che per il madrigale è ormai al termine della propria para-

\section{F. Alberto Gallo, La Polifonia nel Medioevo, cit., p. 71-73.}

39. W. Thomas MARROCCO, "Integrative devices in the music of the Italian Trecento", F. Alberto GALlo (in a cura di), L'Ars nova italiana del Trecento, Certaldo: Centro di Studi sull'Ars nova italiana del Trecento, 1970, (Secondo Convegno Internazionale 17-22 luglio 1969 sotto il patrocinio della Società Internazionale di Musicologia), p. 411-429: 417-418, 424425 e 427; ID. "The newly-discovered Ostiglia Pages of the Vatican Rossi Codex 215: The earliest Italian Ostinato», Acta Musicologica, n. 39/1-2, 1967, p. 84-91. 
bola evolutiva. ${ }^{40}$ Tale varietà nei comportamenti potrebbe essere dovuta non solo a una diversa volontà creativa ma, anzi, induce ad ipotizzare la presenza di linee altre nella trasmissione e penetrazione del Canzoniere petrarchesco sia per la tradizione del testo poetico, sia per quella musicale. ${ }^{41}$ Vie non così laterali, che hanno «sfruttato» il ri-pensamento esercitato dal «calco» e presumibilmente garantito dalla necessità di trovare un "vestito» adeguato, determinando un vigoroso fenomeno di "propagazione» e amplificazione. D'altra parte la distanza temporale, e il fenomeno prospettico che si determina, induce e rafforza l'ipotesi circa la formazione e consolidamento del modello petrarchesco. Ciò, come già osservato, potrebbe in parte aiutare a comprendere il motivo per il quale i musici vicini al Petrarca nelle loro opere sono più lontani dal modello a differenza di Paolo che ormai lo riconosce come tale e cerca il maggior grado di adesione. Ancora il comportamento di Paolo nel madrigale Non più 'nfelice non rassicura che il canale letterario possa essere stato il solo o il principale; infatti la composizione prende a modello musicale la versione di Non al suo amante intonata da Jacopo da Bologna. Tale atteggiamento, peraltro non così infrequente, non smentisce la possibilità che questa sia stata un'altra, non meno importante, strada per la trasmissione dei testi petrarcheschi.

A questo riguardo vi è forse da valutare che nei più tardi anni tra fine Quattrocento e ultimo quarto del secolo seguente, si ebbe la maggior fortuna del Canzoniere presso i musicisti, con un picco di cinquecento madrigali nel 1570 su circa duecento edizioni contenenti almeno un testo del Petrarca. ${ }^{42}$ Quantità di rilievo e, visto il significato anche commerciale che il dato fa spiccare, ulteriormente moltiplica i possibili canali attraverso i quali il canzoniere petrarchesco penetrò e modificò i gusti e l'estetica di un'epoca.

Il dubbio non è relativo ad un canale né vuol stabilire una gerarchia tra i tramiti della trasmissione, e tra questi certamente non manca quello aurale-orale, ma piuttosto tende a sollecitare la questione anche per il Trecento. Resta ad ogni modo aperto anche un altro interrogativo: se la circolazione dei testi del Petrarca, o che ebbero a modello i suoi, che trovarono collocazione nel repertorio polifonico, sia avvenuta attraverso i codici musicali stessi o tramite una dotta committenza, senza la diretta conoscenza, da parte dei musicisti, della sua opera poetica.

40. Cfr. Kelly Stephen Kevin, The works of Nicolò da Perugia, The Ohio state University, Ph.D. 1974, Ann Arbor (Michigan), Xerox University Microfilms, 1975, p. 31-38.

41. Analoga ipotesi è stata sostenuta da Marco GozZI, Il rapporto testo-musica, cit., p. 26-28.

42. Cfr. tesi di laurea di Maria Giovanna Miggiani, "Il Petrarca imbrodolato». Fortuna dei testi petrarcheschi nel madrigale italiano del '500, Università di Venezia, anno accademico 198485; i risultati sono citati anche da Lorenzo BiAnCONI, «Parole e musica. Il Cinquecento e il Seicento", in Letteratura Italiana. Teatro, musica, tradizione dei classici, vol. 6, direzione di Alberto Asor Rosa, Torino: Einaudi, 1986, p. 319-363: 328-332. 

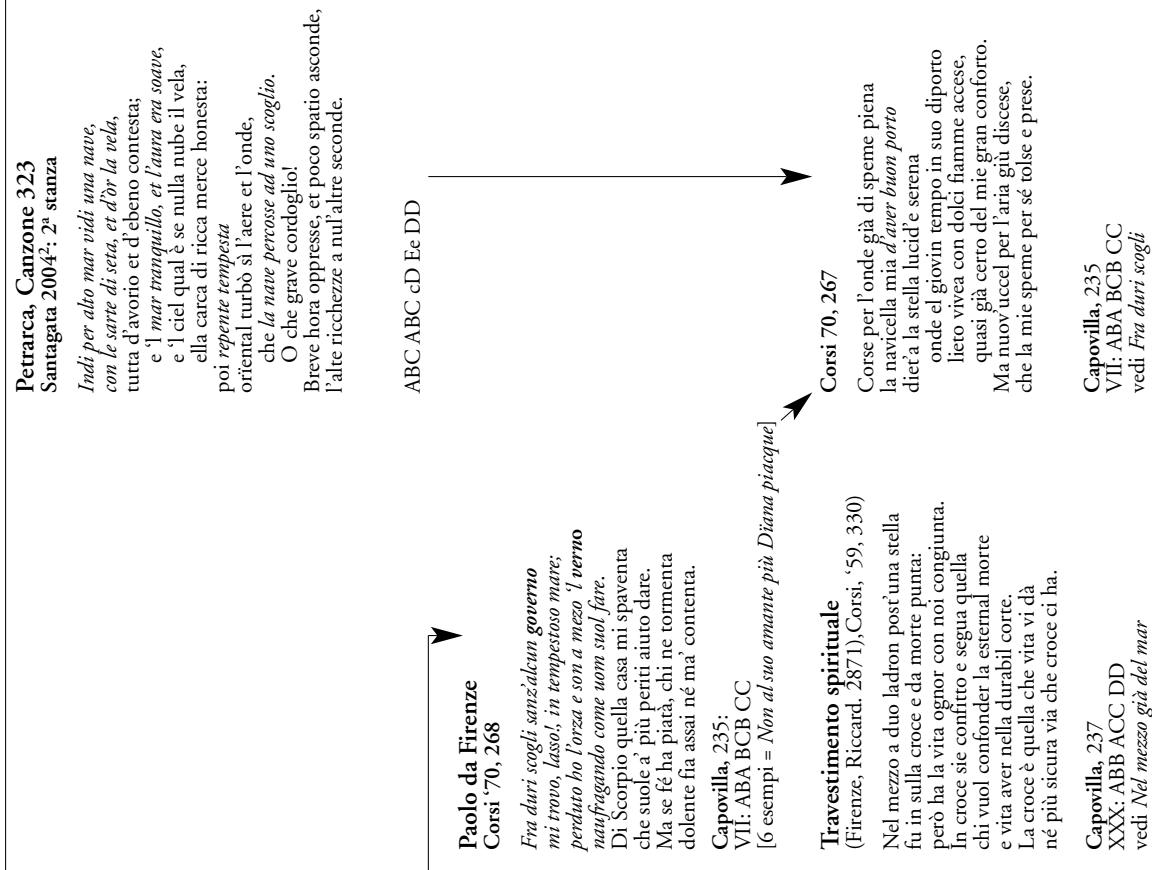

20

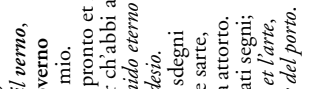

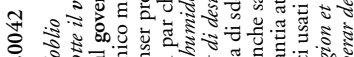

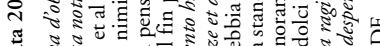

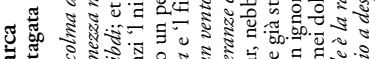

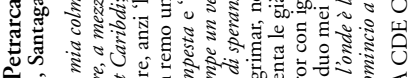
०के ₹ के

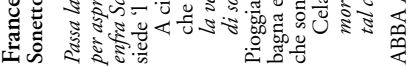

$$
\text { 兽 }
$$

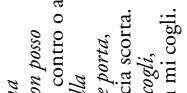

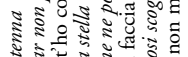
2. है

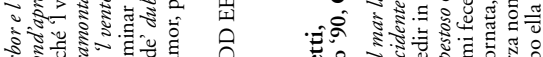

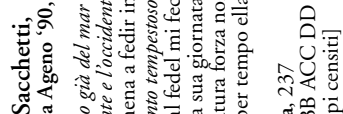
คొ

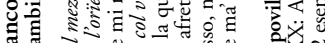

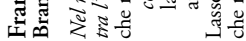

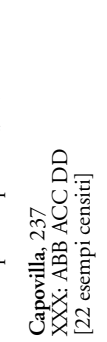




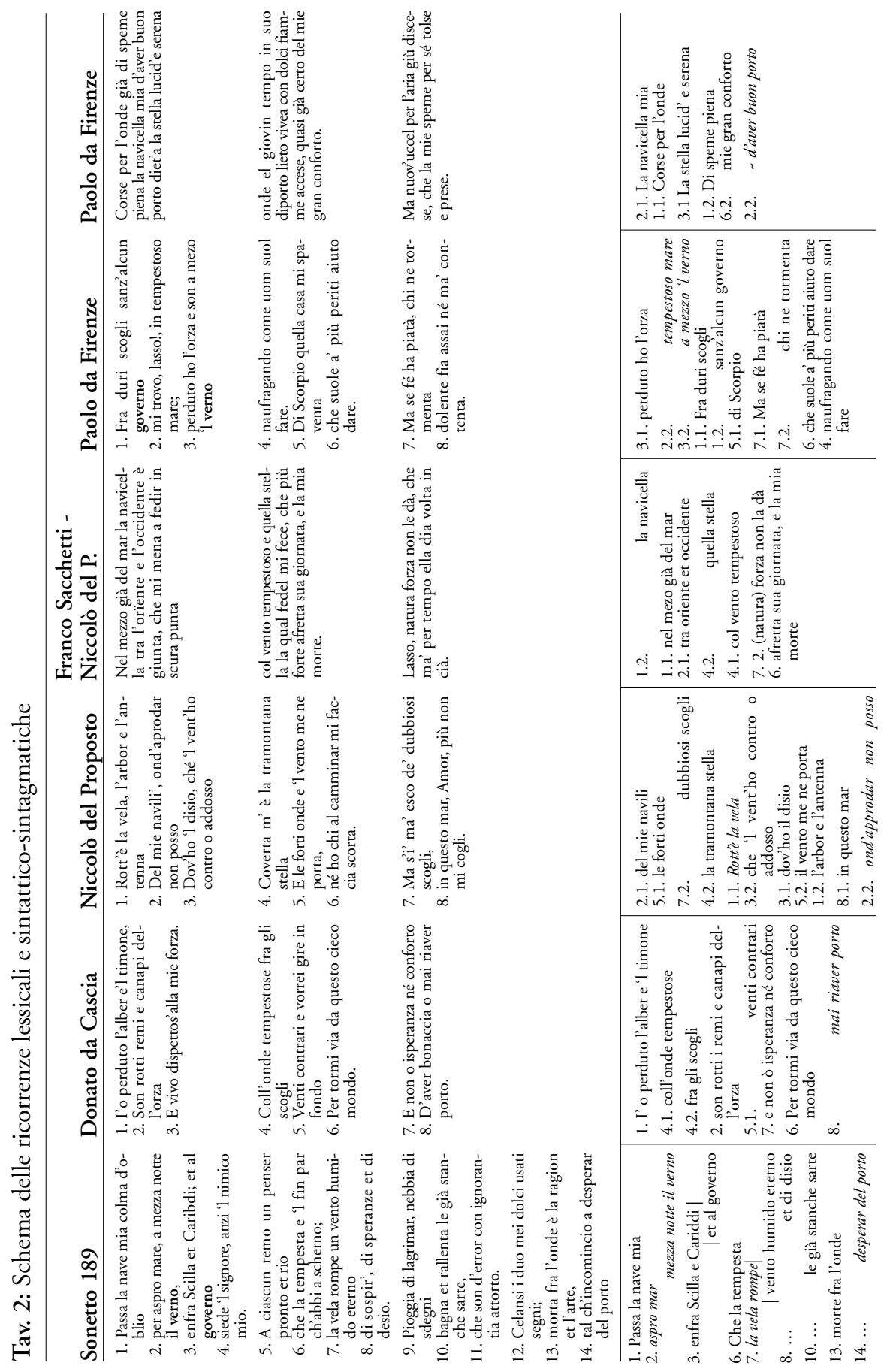




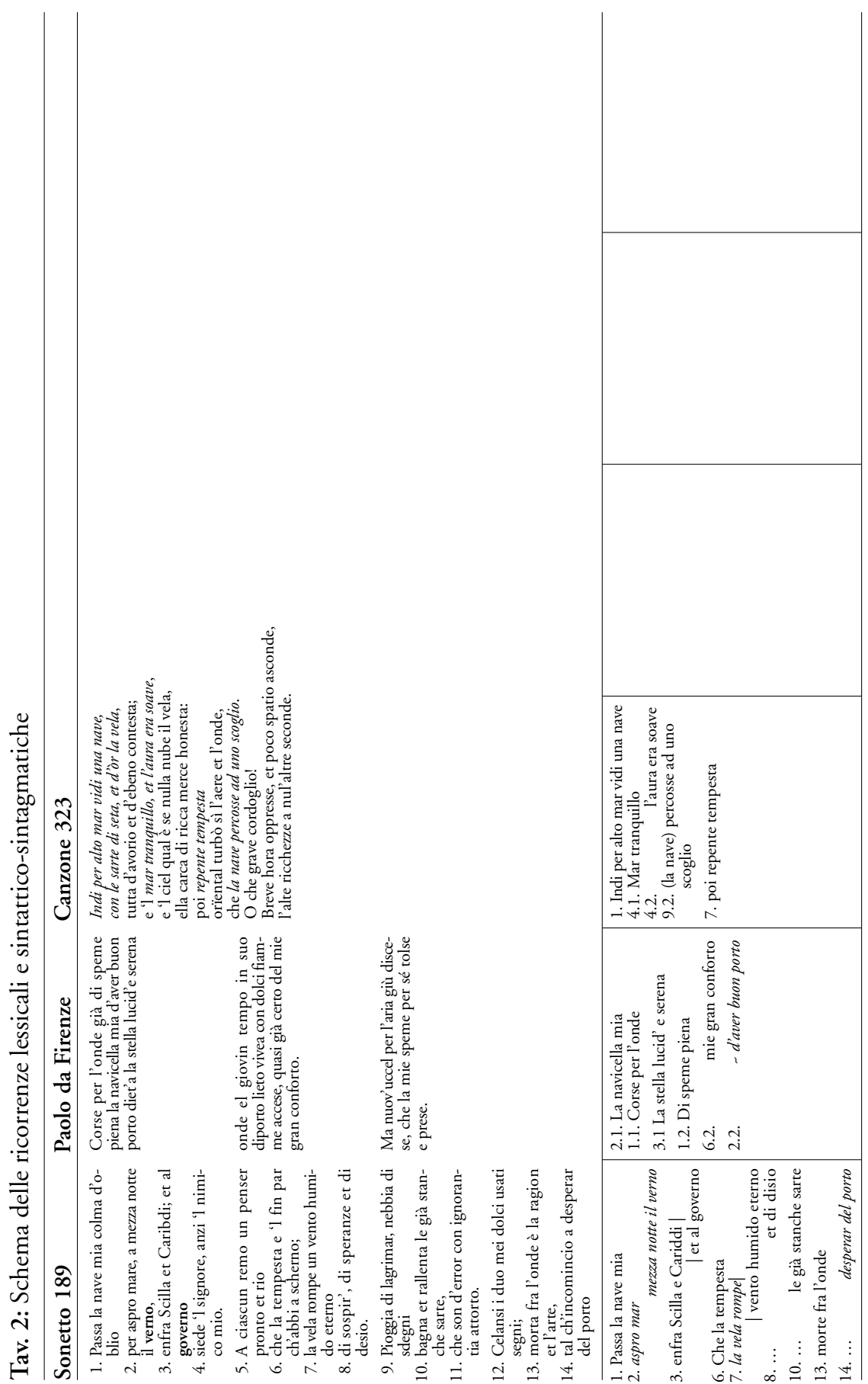




\section{ESEMPI MUSICALI}

Paolo, Fra duri scogli

Es. 1:

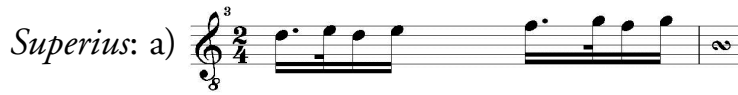

b)

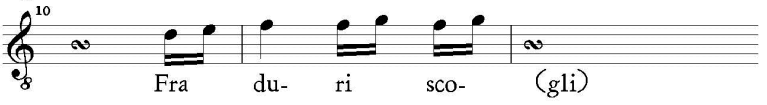

c)

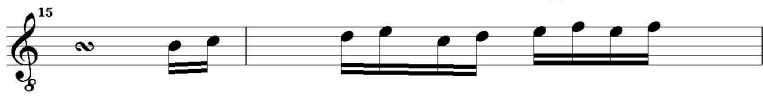

Es. 2

Superius

Tenor

a)

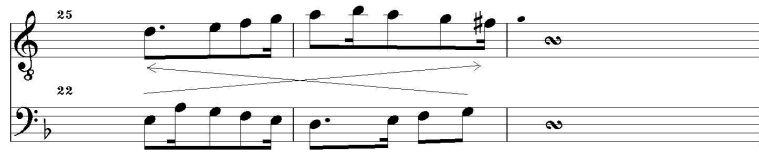

Superius

Tenor

b)

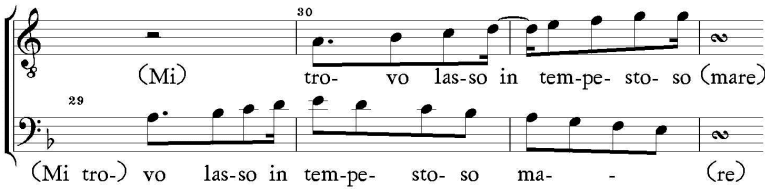

Es. 3

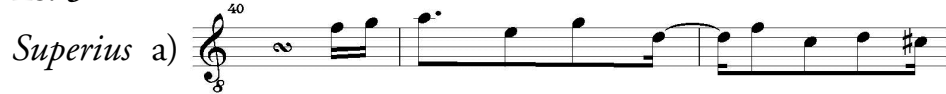

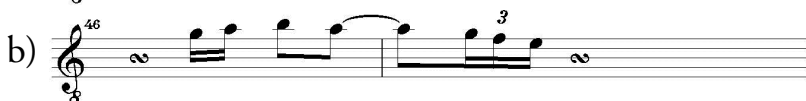




\section{Paolo, Corse per l'onde}

Es. 4

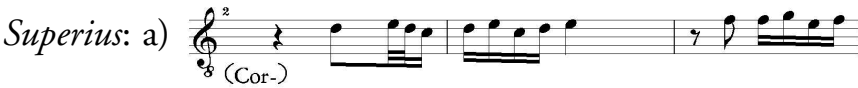

b)

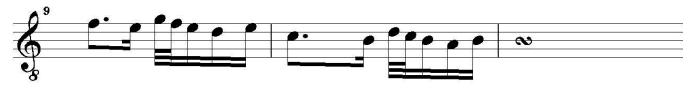

Superius

Tenor

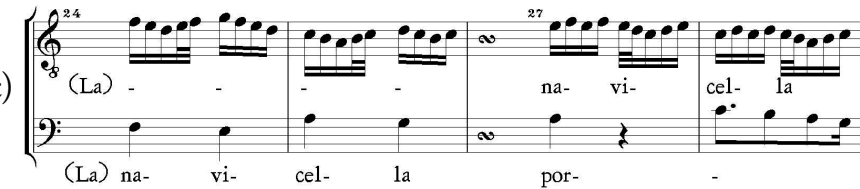

Es. 5

Superius:

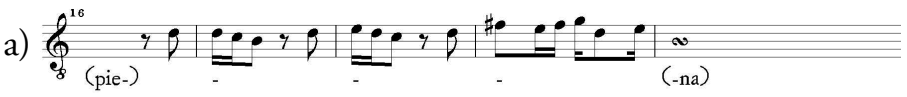

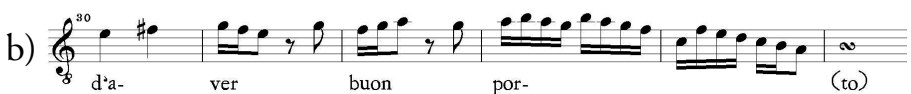

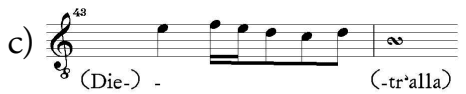

Es. 6

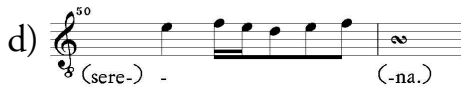

Superius

Tenor
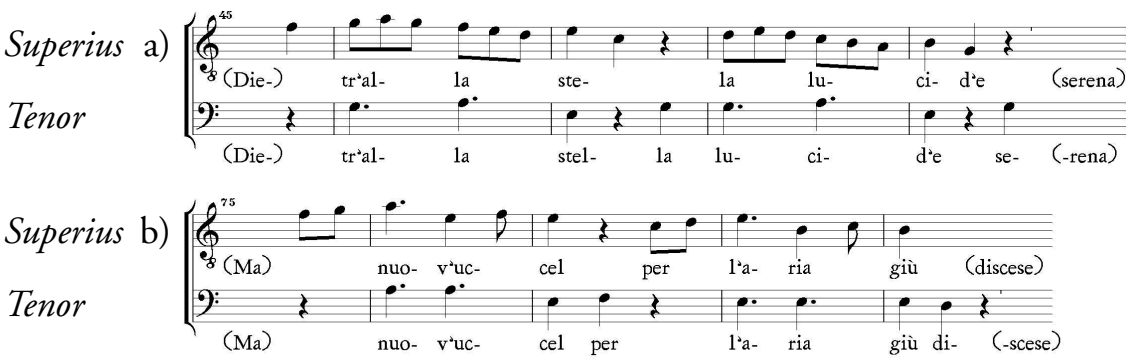

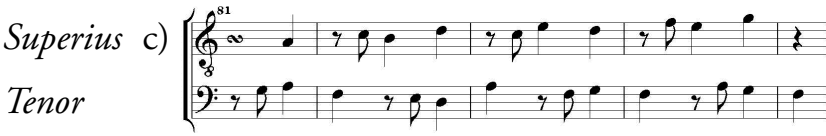

Es. 7

a)

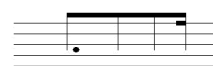

b)

$\overline{\equiv+1+9}$


Nicolò, Nel mezzo già del mar

Es. 8

Superius a) $\ell_{8}^{6} ; \cdot \dot{0} \div \cdots \cdots$

b) $\ell_{8}+\ldots$

c) $\ell_{8} \cdots$

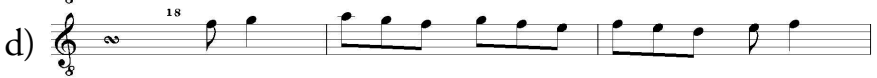

e) $b_{8} \cdots+1 \div \cdots$

f) $\ell_{8} \cdots{ }^{24}$

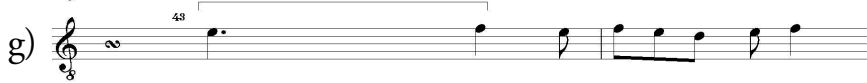

h) $\ell_{8}^{67}, \cdots \cdot ;$

i) $b_{8}^{69}, \quad \cdots \cdots$

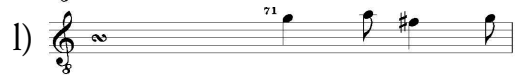

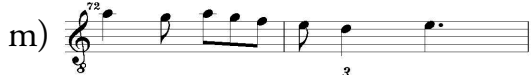

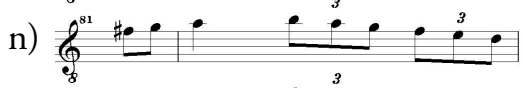

o) $\ell_{8}^{96} \div \bullet \div$

Es. 9

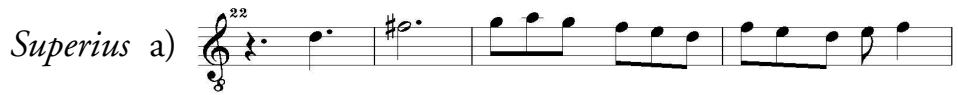

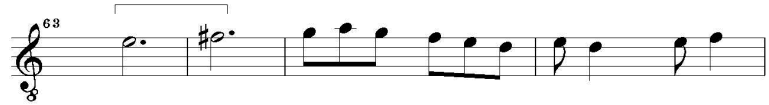

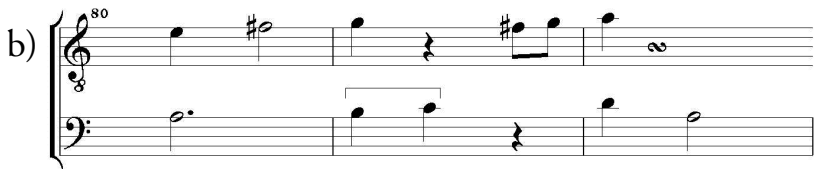

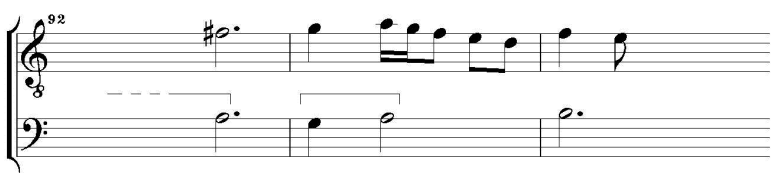




\section{Nicolò, Rottè la vela}

Es. 10

Superius

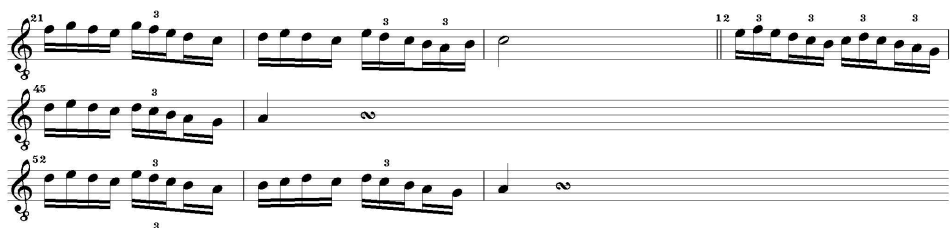

Tenor

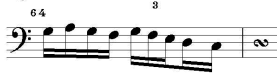

Superius
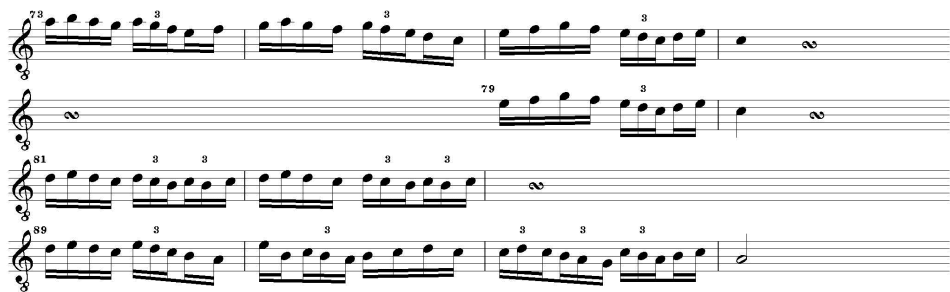

$\overbrace{8}^{14} \omega \cdots \cdots \cdots$

$\overbrace{0}^{43} \infty \cdots \cdots$

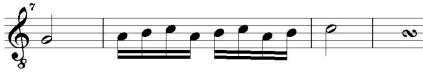

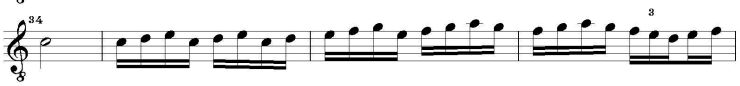

Es. 11

Superius a)

Terzine

Tenor

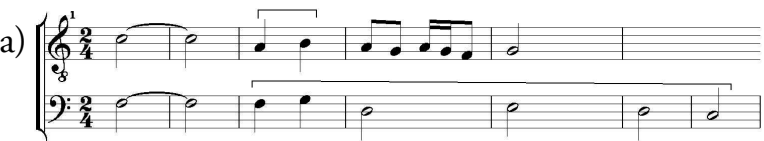

Ritornello

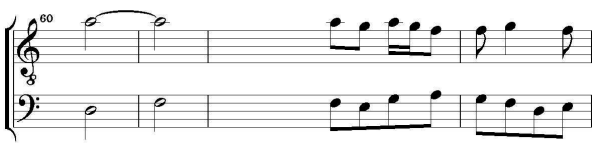

Superius b) Tenor
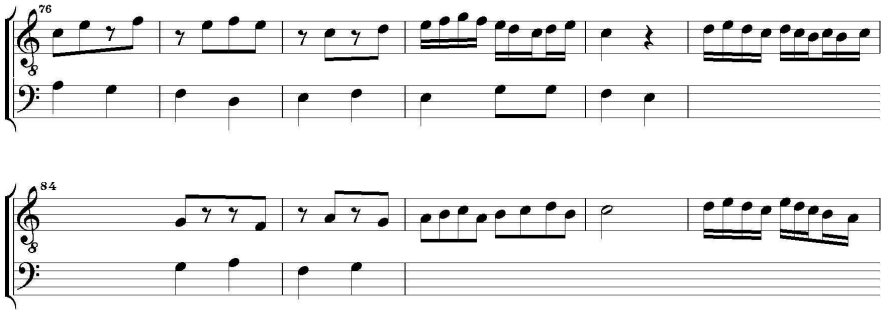


\section{Donato, I’o perduto l'alber}

Es. 12

Superius a)

a) $b_{8}^{5} \cdots, \cdots$
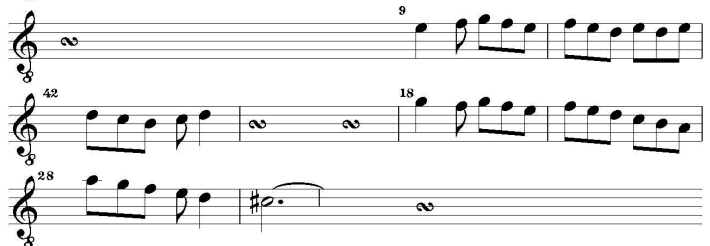

Tenor

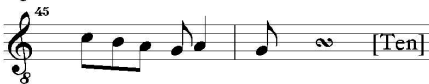

Superius

b) $\oint_{8}^{59} ! \cdots \cdots \cdots$

c) $\ell_{\ell_{8}^{65} \cdots \cdots \cdots}^{\cdots} \cdots \cdots \cdots$

Tenor

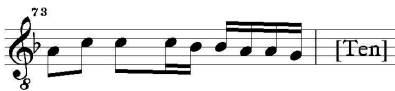

Superius

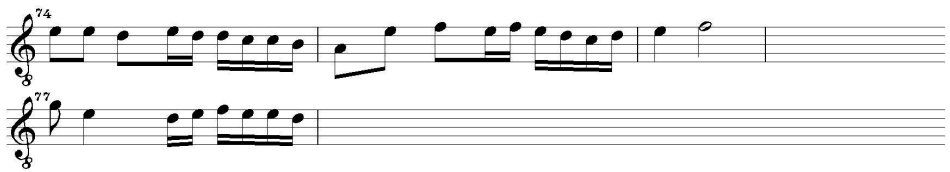

Es. 13

Superius

Tenor
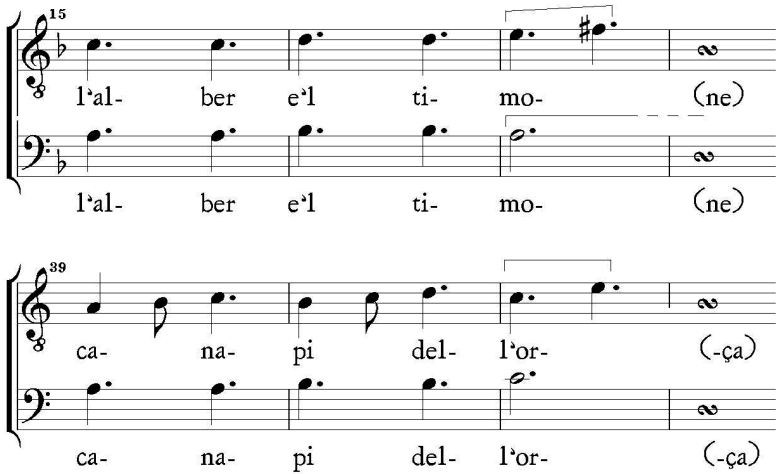
Es. 14

Nicolò,

Rott'e la vela

a)

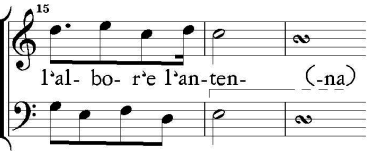

Paolo,

Fra duri scogli
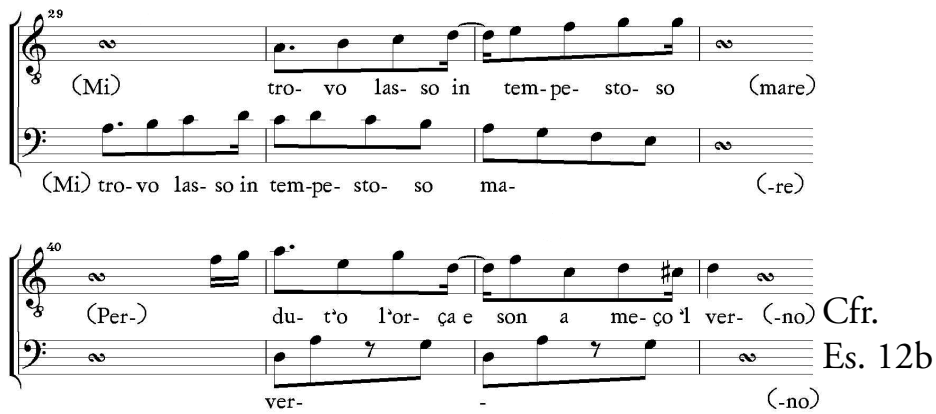

Paolo,

Corse

per l'onde

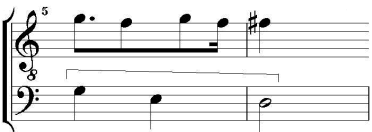

Nicolò,

Rottè la vela

b)

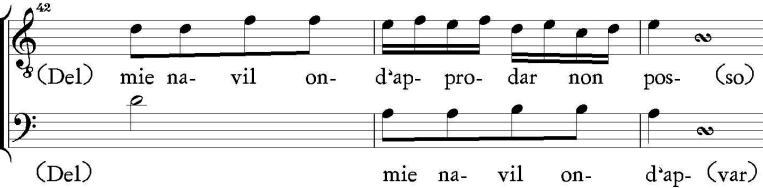

Paolo,

Corse per l'onde

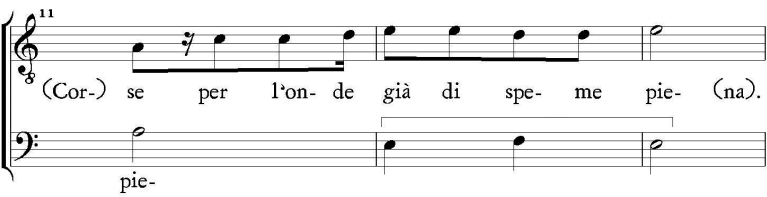


Es. 15

Paolo, Corse per l'onde

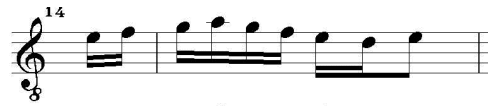

Nicolò, Rott'è la vela es

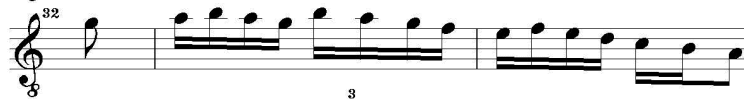

Es. $11 \mathrm{a}$

Es. 16

Paolo, Fra duri scogli

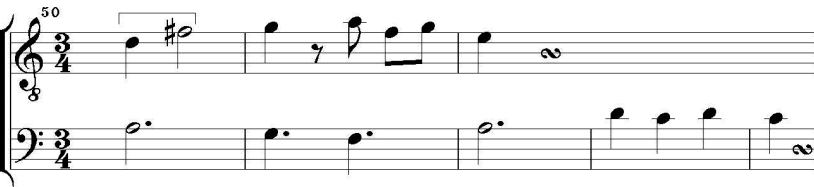

Nicolò, Nel meço già

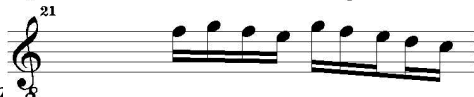
Cfr.

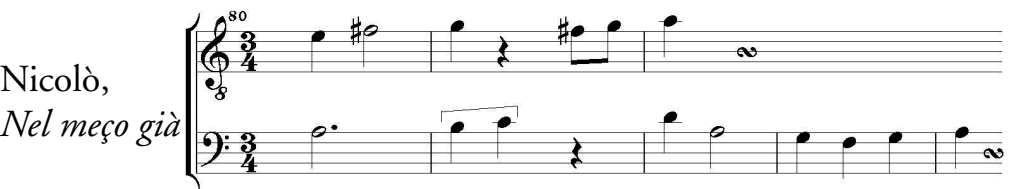

Es. 17

Donato, I'o perduto

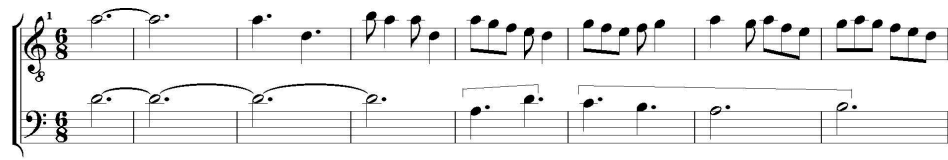

Nicolò, Nel meço già

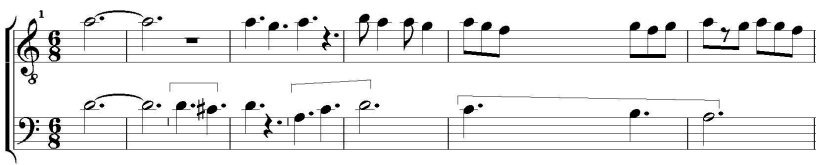

Es. 18

Donato, I'o perduto

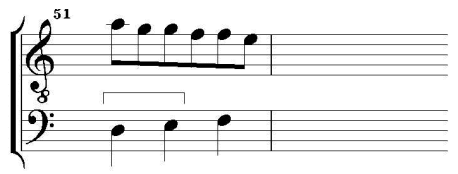

Paolo, Corse per l'onde

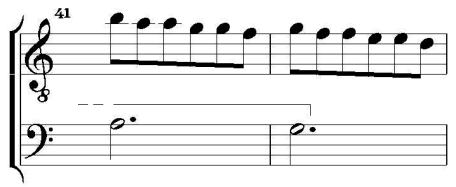

